\title{
Analysis of Electromagnetic Disturbances Generated by an Autonomous RFID-Based Navigation Robot
}

\author{
Marcin Hubacz¹, Dariusz Klepacki², Kazimierz Kuryło², Bartosz Pawłowicz², Wiesław Sabat² ${ }^{\star}$ \\ Bartosz Trybus ${ }^{3}$

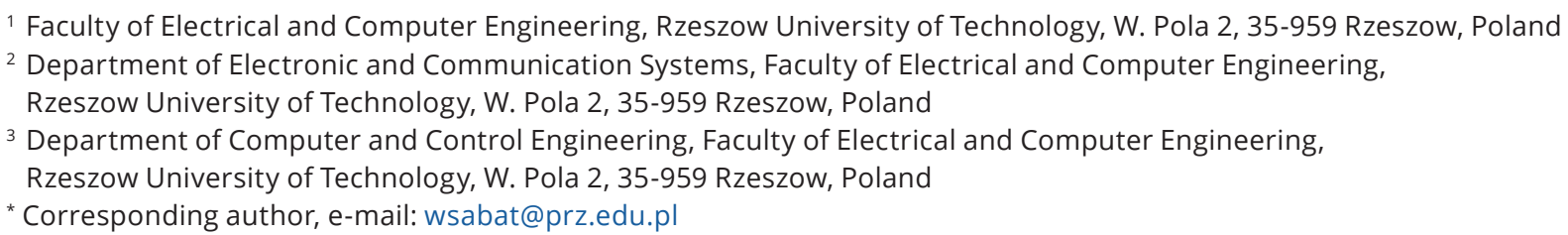

Received: 15 November 2018, Accepted: 08 January 2019, Published online: 19 March 2019

\begin{abstract}
The paper discusses the issue of EMC compatibility of autonomous navigation robots that utilize the RFID technology for surface discovery. The robots can work individually or in a group in environments equipped with RFID transponders. The need to examine EMC compatibility of such robots is pointed out, so that they can work and communicate without disturbing other devices or each other. Measurements and analysis of electromagnetic disturbances generated by a prototype of a navigation mobile robot has been carried out. Results of the measurements for the model of the robot are presented. Factors determining generation of the disturbances in the model are pointed out.
\end{abstract}

Keywords

EMC, RFID, mobile robot, group of robots, surface exploring

\section{Introduction}

The introduction of automatic identification systems for contactless RFID (Radio Frequency IDentification) brings a number of advantages. They are used to identify objects with passive or semi-passive transponders (or tags) equipped with a writable memory with an information capacity definitely exceeding currently used barcodes [1]. RFID system may be used in many areas, especially in the field of ISM (Industrial, Scientific, Medical).

The important advantage of RFID systems is that it is possible to read information from many objects with usage of anticollision protocols. A robot equipped with an RFID reader can use information and coordinates stored in RFID transponders built in walls, floors, doors and furniture [2]. Unfortunately, reading the information does not give knowledge about the location of an identified object on the surface or in the space. For this reason, quickly reaching the right object can be a problem. Hence, in the scope of RFID systems, the problem of location of an object on which the transponder is mounted, is an important research area [3-8].
It was also noticed that it is possible to reverse this issue and attempt to determine the position of a mobile robot equipped with an RFID reader based on readings and analysis of signals received during readout of transponders placed in known locations [9-11]. Many of the existing studies are based on the application of the RSSI method (received signal strength indicator) [11, 12]. This is sometimes extended with methods related to odometry and application of EKF filtering to increase the accuracy of position estimation [13]. There are also original solutions based, e.g., on phase measurement of the signal reflected from the transponder and carrying the information read from the transponder [14].

However, the mentioned studies mostly feature a very extensive mathematical apparatus and almost completely ignore the issue of orientation and localization support with data stored in the transponder's memory. This gap was quickly noticed [2]. It resulted in development of various solutions using RFID transponders to determine the location and orientation of a robot equipped with an RFID 
reader, starting from simple solutions [15], to advanced systems using the power measurement of the signal reflected from RFID transponders arranged in a grid [16-18]. It is significant, however, that due to interference [19], the most commonly used solution is the installation of a single reader in a mobile robot.

Currently, one of the most interesting and promising research topics are "intelligent" autonomous robots intended for indoor mapping. The main functions of this type of robots is to gain knowledge about the surface on which the robot moves or to navigate the robot to a designated point. Applications of such robots are miscellaneous, ranging from automated warehouses of goods, helping people in wheelchairs or the blind, up to simply bringing coffee from the kitchen to the dining room. It is worth noting that the area or surface the robot moves on may be reconfigured from time to time (e.g. by installing new shelves in the warehouse). Hence, the robot-based automated recognition does not have to be a one-off process, but the discovery should be carried out periodically to detect the changes.

The RFID technique [1, 20, 21] is used often in such solutions as a part of the robot navigation system. In this concept, the robot equipped with one or more RFID readers map an indoor area using information and coordinates stored in a grid of RFID transponders. In addition, the transponders may hold extra information about the type of terrain in its neighborhood. Another option is employing multiple robots (a swarm) for more efficient surface mapping. The robots communicate with a host or with each other. For these reasons, the robot can be treated as a radio communication device [22].

The aspect of mutual communication between robots in the swarm requires providing a clean space in the electromagnetic sense. This space should have a standard level of generated electromagnetic disturbances. The level of generated disturbances has a significant impact on the sensitivity of devices transmitting information in the system, especially in the case of the HF range (high frequency), such as RFID. If the level of disturbances reaching the receiver exceeds its level of sensitivity, the system may not function properly and interpret erroneously the information transmitted in the system. These disturbances can be generated by the robot itself, other robots moving nearby, as well as electrical and electronic devices not belonging to the system.

A potential source of electromagnetic disturbances in the navigation robot is its propulsion system. In modern solutions, PMDC brush motors, BLDC brushless and step motors are most often used to drive a robot. In the case of motors with the classic commutator, the process of commutation of energy in the windings is accompanied by the effect of generating electromagnetic disturbances. These disturbances are characterized by wide harmonic components and their stochastic allocation in the spectrum. In the case of BLDC electronic circuit-switched motors, the harmonic components generated in the spectrum are static, but the spectrum is also broadband. The range of harmonics in the spectrum, their position and number are determined by the motor parameters, its rotational speed and control technique.

Another potential source of disturbances is the motor control system and the power supply circuit. The necessity to reduce thermal losses in keying circuit forces the designer to increase the switching speed. In the aspect of electromagnetic compatibility, this procedure may lead to an intensification of disturbances generated by the system.

Each autonomous robot includes one or more specialized microcontrollers. They control its functions and process data generated and acquired by the robot. In the case of mobile navigation robots, the information must be collected and processed very fast using microcontrollers of adequate performance. The microcontrollers applied in control systems (usually 16- and 32-bit) are potential sources of disturbances. The spectrum generated by these elements is predictable, but it changes dynamically depending on the microcontroller operating state. The harmonic components generated by clocks, clock buses and external interfaces handled by the microcontroller may have a significant level. Requirements in this area are defined in the EMC Directive 2014/30/WE and a series of harmonized standards [23]. The requirements for devices are specified in the standards according to the environment in which the device works, as well as its category. In the case of the EMC directive, an important aspect is the assignment of the tested device to the target working environment. Hence, the environment in which the object operates and the type of device determines the allowed level of disturbances that it can generate.

A device that contains radio equipment is a subject to the RED radio directive [24], which protects the radio spectrum. Requirements in this area are specified in the ETSI standards. It is obligatory to meet the requirements of the EMC and RED directives. In the case of the HF RFID system, the reader generates the $13.56 \mathrm{MHz}$ signal. The information is returned back to the system using the $200 \mathrm{kHz}$ channel. 
In this paper, a prototype robot is considered, which does not have a specialized standard in the field of electromagnetic compatibility, so it will be analyzed in accordance with the requirements of the EN 61000-6-3 standard and its worldwide counterpart [25]. This standard specifies the levels of disturbances and techniques of their measurements for devices dedicated to work in residential, commercial and slightly industrialized environments.

By analyzing thematic studies in scientific databases one can realize that the subject of electromagnetic compatibility of mobile robots is very actual and requires consideration of a number of issues.

\section{Robot construction}

The prototype of the laboratory navigation robot used in the conducted tests is a class 2.0 construction. It is a nonholonomic robot with two non-steering, independent drive wheels and two optional supporting points. The simplified construction allows for easy and high efficient movements, using small number of elements and motors.

The components of the navigation robot are shown in Fig. 2. The robot may be equipped with one, two or four RFID readers with antennas. The option with two readers is depicted in Fig. 1. Each reader has its own antenna working with a different channel within the selected frequency range to avoid interference [19]. Data from all transponders located within the RFID range should be reflected [1].

The surface area examined by the robot is covered with a grid of RFID transponders. Each transponder has its own identification data denoting its position in the grid. It is assumed, that the robot is able to properly determine its location on the surface using the information gathered by its readers from the transponders located on the surface.

By using multiple readers in a robot, we can improve computing the location of the robot and the distance between a reader and a transponder. If only one antenna is considered (Fig. 2a)), the robot will not be able to recognize the direction it follows. In addition, data stream read from the tags it passes by is hard to interpret. As the result, one cannot use data from neighbor tags and the main asset of RFID grid is lost. In order to properly determine the relation between the tags, the robot should have at least two antennas (Fig. 2b). It will then be able to recognize the two basic movement directions, i.e. "forwards" and "backwards". However, only four antennas will give the full information, including the two other directions ("right", "left").

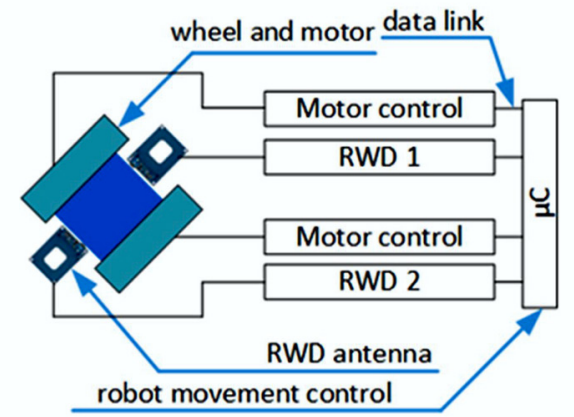

Fig 1 Components of a robot with RFID-based navigation a)

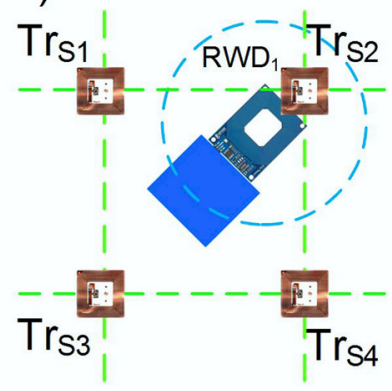

b)

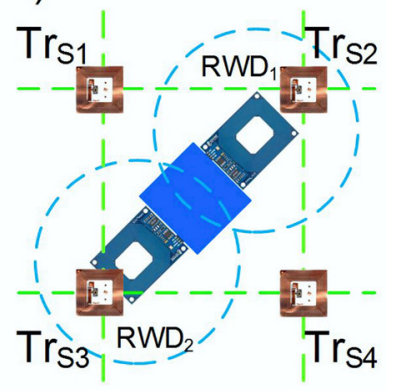

Fig. 2 The navigation robot with: a) one RFID reader, b) two readers

Using the RFID technology for robot navigation requires to select the frequency range and to configure position of antennas and readers in a robot. In the proposed solution, we decided to use HF range [1] and well-documented ISO 15693 and ISO 14443 communication protocols [20, 21]. The selected standards seem appropriate for indoor applications with up to about 50-60 centimeters of RFID reading range $[26,27]$.

The laboratory prototype of the navigation robot is shown in Fig. 3. The electronics is built based on STM32F10RBT6 (ARM Cortex-M3 core) from STMicroelectronics, 128kB of flash memory and 20kB of RAM. The RFID reader with PCB antenna is based on MRC522 chip from NXP. The chip gives multiple connection options with the main $\mu \mathrm{C}$, but we have used SPI which seems appropriate to handle multiple readers in the future.

Control of the independent drive wheels with DC brush motors is done via H-Bridge motor driver TB6612FNG from Toshiba. Each of the wheels is simultaneously PID controlled with feedback from encoders located on the motor shaft. The robot has also been equipped with four distance sensors located at the front. Analog sensors are based on LIRT5B phototransistor and IR SFH4550 diodes. 


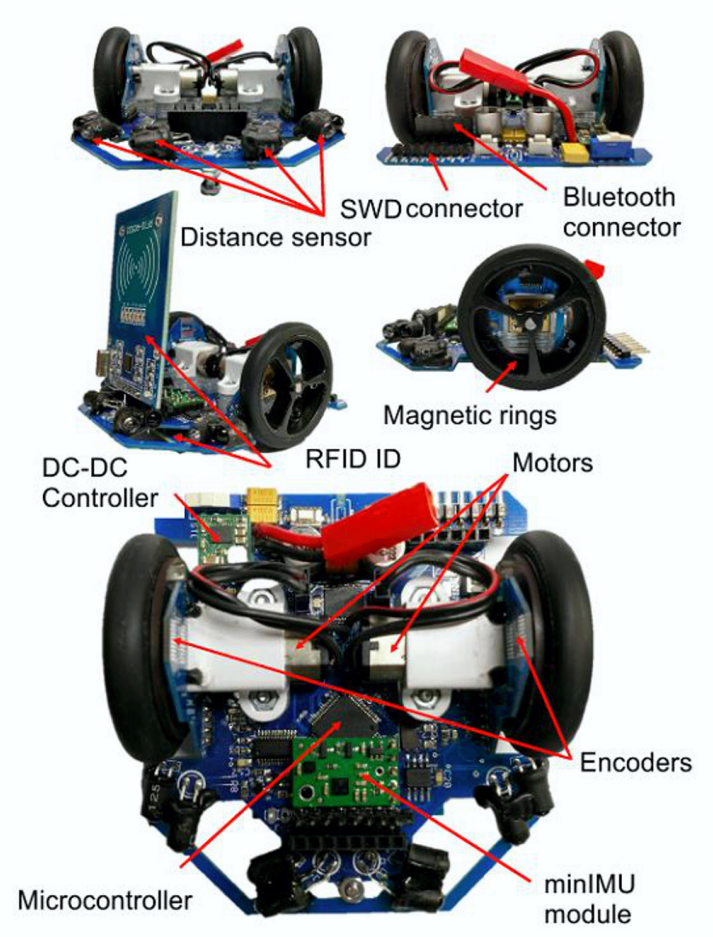

Fig. 3 View of the laboratory robot prototype

\section{Measuring the radiated emission of the robot prototype}

Evaluation of conformity with the EMC Directive recommendations of an electrical and electronic device requires the determination of its emission level and resistance to standard types of electromagnetic disturbances [23]. According to the provisions contained in the standards, the so-called conducted emission from power and communication interfaces is examined. It is set in the frequency range from $150 \mathrm{kHz}$ to $30 \mathrm{MHz}$ for most civilian objects. For some other objects, e.g. elements of lighting or medical equipment, the analysis is carried out from $9 \mathrm{kHz}$. The radiated emission is determined in the frequency range from $30 \mathrm{MHz}$ to $6 \mathrm{GHz}$. The upper frequency value depends on the operating frequency used in the examined system. If the frequency of clock signals in the system does not exceed $108 \mathrm{MHz}$, the analysis may be limited to $1 \mathrm{GHz}$.

The measurement of both conductive and radiated emissions should be carried out in a space free of electromagnetic disturbances. Due to the lack of such places in the urbanized world, the measurement is usually performed in the Semi-Anechoic Chamber (SAC) with conductive floor, simulating conditions similar to a free space with conductive ground. The measurements of the robot prototype presented in this paper were carried out in the TDK anechoic chamber in the Electromagnetic Compatibility Laboratory of the Department of Electronic and Telecommunications Systems of Rzeszów University of Technology.

As said, there are no suitable standards for the examined robot. Therefore, its emission has been tested in accordance with the European EN 61000-6-3:2008 and EN 61000-6-3/A1:2012 standards. These are general standards and their recommendations fit a wide range of electrical and electronic components that generate disturbances and are susceptible to them.

The examined robot is an autonomous object and it uses a battery as the energy storage device during normal operation. So, it is not necessary to measure the conducted emission unless it is conditioned by internal compatibility. However, the level of radiated emission must be determined.

In order to make the tested object work in conditions close to reality, the robot motors were loaded similarly as when moving on a real surface. For the measuring purposes, an adjustable brake was built. During the tests the examined robot was in motion and the brake controlled the load of the motors (Fig. 4). The robot with the brake was placed on a rotating table $0.8 \mathrm{~m}$ high.

According to the standard, the object was rotated by $360^{\circ}$ during the test and the measuring antenna was changing its position from $1 \mathrm{~m}$ to $4 \mathrm{~m}$ with the step no greater than $1 \mathrm{~m}$. The object was positioned in a distance of $3 \mathrm{~m}$ from the measuring antenna. This way of moving the object allows to find all potential places and directions of the radiated disturbances.

The level of emission of disturbances is measured in $\mathrm{dB} \mu \mathrm{V} / \mathrm{m}$. A receiver with a built-in peak, quasi-peak and average peak detector and a set of antennas is used for this purpose. The measured values are referred to the boundary values contained in the EN 61000-6-3 standard. During the tests of the robot, the $R \& S^{\circledR}$ ESU26 receiver and the $R \& S^{\circledR} H K-116$ and $R \& S^{\circledR}$ HL-223 antennas from Rohde \& Schwarz were used.

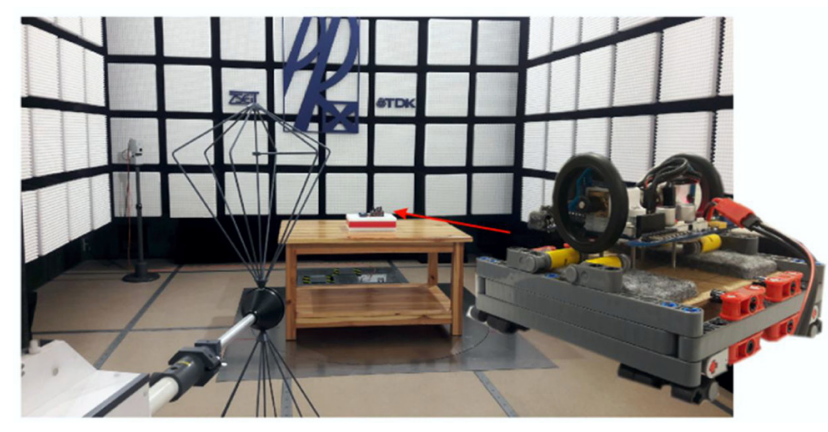

Fig. 4 Configuration of the measurement bench inside the anechoic chamber and the tested object along with the brake 
The dynamic changes of the location of the tested object, the height of the suspension of the antenna and its polarization, as well as the scan of the spectrum for each position of the antenna and the object requires the use of specialized software for data acquisition and analysis. Manual analysis is not possible due to over 100,000 measurement points considered in the procedure. The quantity of measurement points is determined by the step with which the frequency at the receiver is changed and the number of the table and antenna positions. The antenna polarization is also taken into account. To automate the measurements, the $R \& S^{\circledast}$ EMC32 software from Rohde \& Schwarz has been used in the tests. Its structure allows for flexible configuration and change of measurement configuration scripts. In order to investigate the conformity of the prototype robot with the recommendations of the standards, a measurement script was created (Fig. 5) to analyze the emission of the radiated disturbances.

The measurement of disturbances was carried out in the frequency range from $30 \mathrm{MHz}$ to $1 \mathrm{GHz}$. As it is recommended in the standard, a preliminary, quick assessment of disturbances was carried out using a peak detector. A quasi-peak value detector was used for critical values. The value of the measurement frequency was changed with the step of $\Delta f=40 \mathrm{kHz}$ within the range of the analyzed frequencies. This value is related to the width of the filter used for the measurement, i.e. $120 \mathrm{kHz} / 6 \mathrm{~dB}$. According to the measurement technique, the step of the measurement frequency cannot be greater than half of the width of the filter. In addition, in the case of the quasi-peak detector, whose measurement is conclusive, it is required to hold the measurement for a period of minimum 1s for each measurement point.

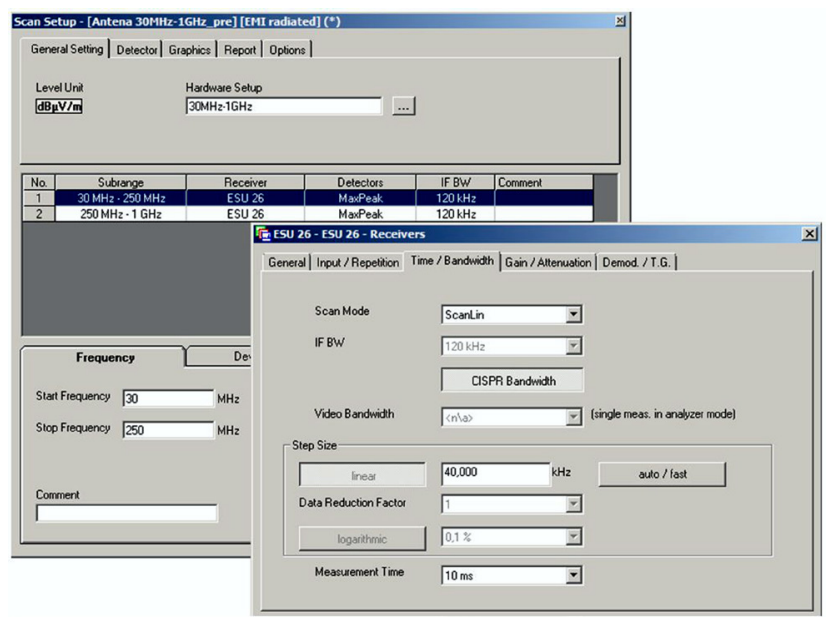

Fig. 5 Script for measurement of radiated emission in $R \& S^{\circledR}$ EMC32 software
The measurements were carried out for three characteristic speeds of the robot, i.e. $0.1,0.5$ and $1 \mathrm{~m} / \mathrm{s}$ accordingly.

The analysis of the disturbances generated by the examined robot showed that regardless of the speed of its movement, it meets the requirements of the standard. However, in the case of the highest speed, the critical value of the disturbance level is $2 \mathrm{~dB}$ lower than the allowed value. The speed itself has undoubtedly a measurable effect on the level of generated disturbances. As seen in the waveforms shown in Figs. 6-8, if the speed drops, the level of disturbances also decreases. The waveforms are presented separately for vertical and horizontal polarization of the measuring antenna. The larger level of disturbances for horizontal polarization is determined by the PCB arrangement in the robot assembly. As will be confirmed in the next chapter, the disturbances are conditioned by the propulsion system.

The process of speed control that utilize PWM modulation technique and commutation of energy on the commutator is the source of increased emission in the frequency

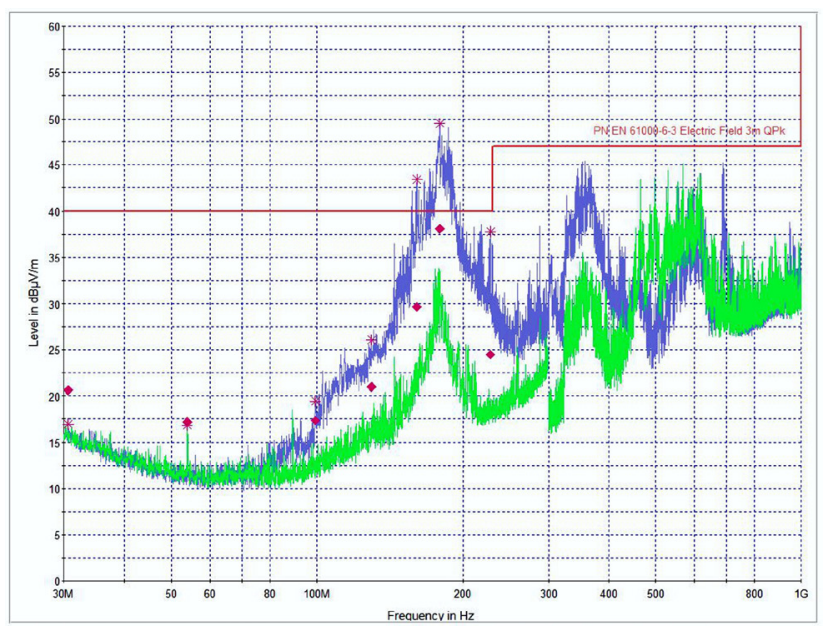

Fig. 6 Results of measurements for the speed of $1 \mathrm{~m} / \mathrm{s}$.

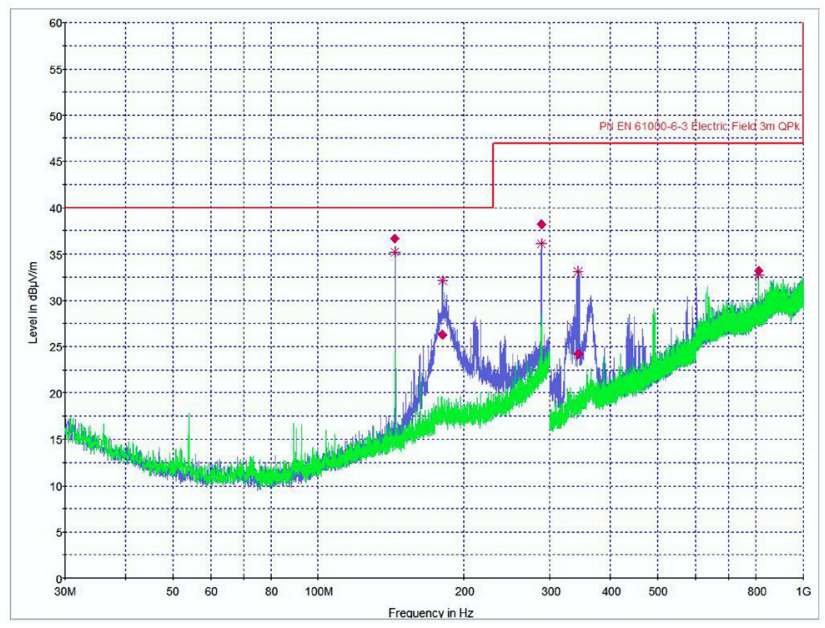

Fig. 7 Results of measurements for the speed of $0.5 \mathrm{~m} / \mathrm{s}$. 


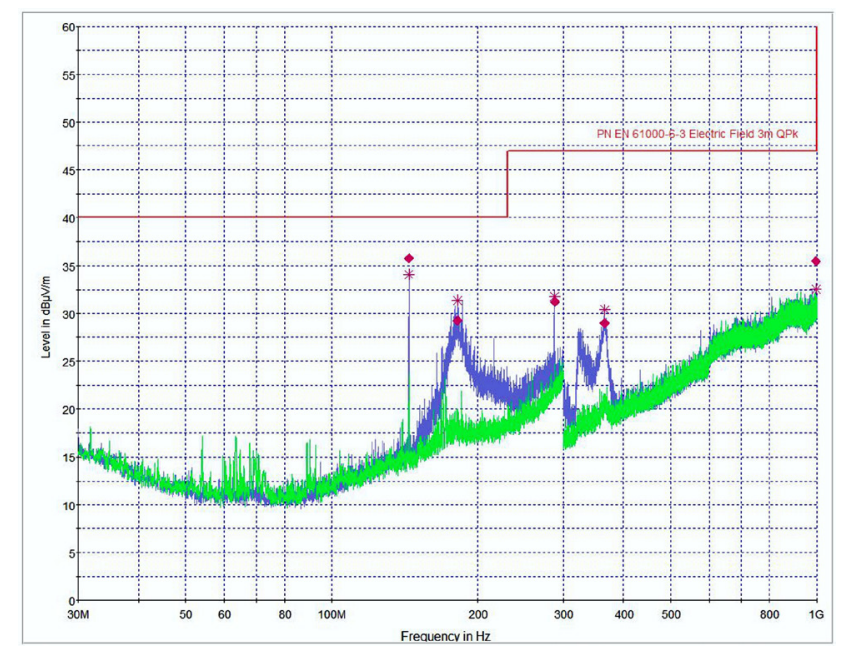

Fig. 8 Results of measurements for the speed of $0.1 \mathrm{~m} / \mathrm{s}$.

band from 100 to $800 \mathrm{MHz}$. At lower speeds in the spectrum, the harmonic of the processor clock and its multiples has a significant part.

Taking into account that the speed of the robot's movement has a measurable impact on the level of emitted electromagnetic disturbances, it was decided that in the second stage of research the shape of the emission levels will be determined during robot movement on the test surface. The specifics of the emission measurement require that the object under test should be located in the antenna measurement field. This requirement limits the robot's movement space during testing. In order for the robot to be able to move and simultaneously be in the antenna's measuring field during measurements, a test platform was made. During the measurements, the robot's movement was limited to circular motion. The built platform allowed to determine its emission in real conditions.

As in the case of robot work on the adjustable brake, the analysis was carried out for three representative robot travel speeds, ie $1,0.5$ and $0.1 \mathrm{~m} / \mathrm{s}$. The analysis of the emission of disturbances generated by the robot in the conditions of its tethered motion showed a limited correlation of spectra. The characteristic resonant point in the $180 \mathrm{MHz}$ band is the effect of the radiation of the cable connecting the robot with the battery. In the case of operating with the adjustable brake, this cable was laid horizontally. In the spectrum for this case, the dominance of the harmonic components for the horizontal position of the measurement antenna can be clearly seen (Fig. 6-8). In the case of robot movement on the platform in this frequency band, the harmonic components for the horizontal and vertical polarization of the antenna have similar levels (Fig. 9).

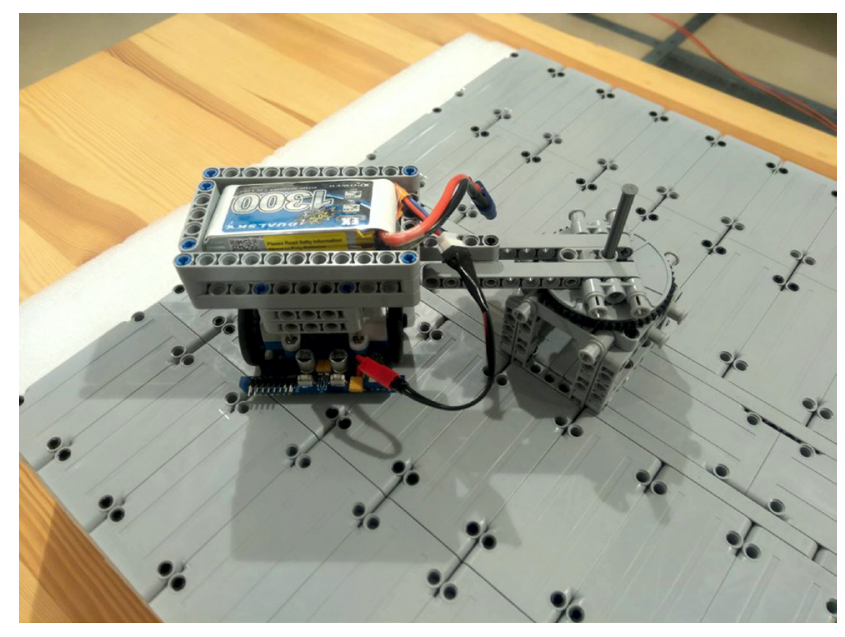

Fig. 9 A photograph of a platform for measuring the emission of electromagnetic disturbances generated by a robot in a state of motion

This state justifies the horizontal arrangement of PCB with robot electronic circuits and vertical wiring connecting the robot with the battery (Figs. 10, 11).

Comparing the measurement results for the brake and platform, it can be seen that in the case of robot movement on the surface additional harmonic components in the frequency range up to $6 \mathrm{GHz}$ appeared in the spectrum (Fig. 12). This condition can be justified by high engine speed and the commutation process of energy on the commutator sections. Additionally, in the case of the adjustable brake, the movement of the wheels was uniform. In the case of movement on the platform's surface, the vibration effect was noticeable at the highest speed. The dynamic load changing to a certain extent when moving on the surface leads to a change in commutation conditions and the generation of additional harmonic components. Taking into account the requirements of the EN61000-6-3 standard and referring to the frequency

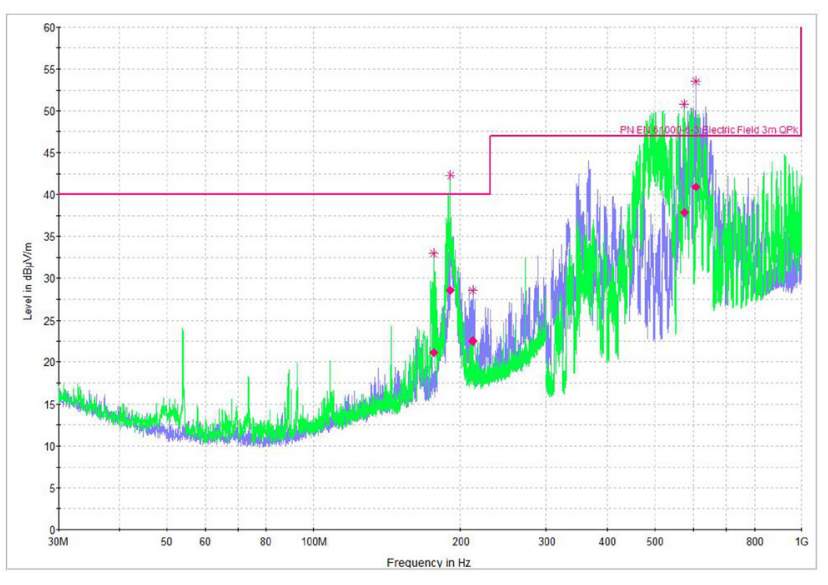

Fig. 10 The results of emission measurements in the frequency range from $30 \mathrm{MHz}$ to $1 \mathrm{GHz}$ for the robot's speed equal to $1 \mathrm{~m} / \mathrm{s}$ 
of clocks clocking in the system ( $\max 24 \mathrm{MHz}$ ), the emission measurement could be limited to $1 \mathrm{GHz}$. However, as shown by the measurements carried out in the frequency range from $1 \mathrm{GHz}$ to $6 \mathrm{GHz}$ (Fig. 12), the presence of harmonic components in this frequency range shows that the frequency of clocks cannot be the only criterion determining the value of the upper measurement frequency.

\section{Factors determining radio disturbances in the robot}

If the disturbances exceed the allowed level or the distance to the acceptable value is small, faces the dilemma of the answer to the reason. Certain characteristic values of harmonic components in the spectrum may suggest elements of the system responsible for this state. To confirm the hypotheses, a set of universal measuring instruments can be used, such as an oscilloscope, a spectrum analyzer equipped with probes of the near field, or current probes.

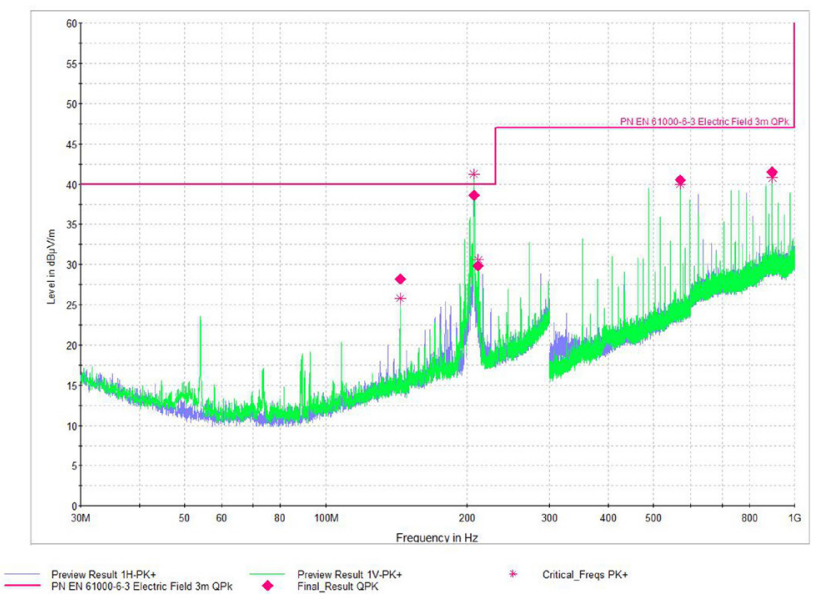

Fig. 11 The results of the radiated disturbances measurement in the frequency range from $30 \mathrm{MHz}$ to $1 \mathrm{GHz}$ for $0.1 \mathrm{~m} / \mathrm{s}$ robot's speed

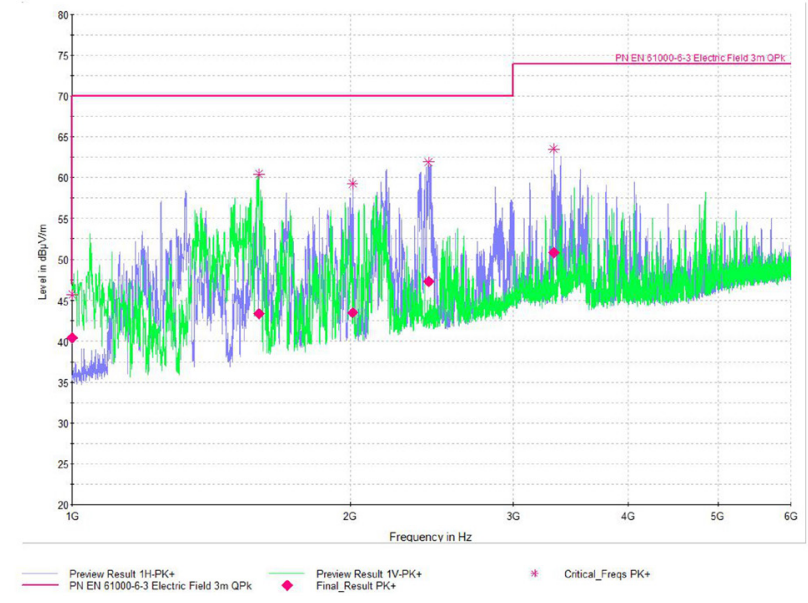

Fig. 12 Measurement results of radiated disturbances for a speed of robot equal to $1 \mathrm{~m} / \mathrm{s}$.
Disturbances emitted by a tested object in accordance with the requirements of the standards are determined for strictly defined distances of 3,10 or $30 \mathrm{~m}$. Electromagnetic waves propagating over these distances are absorbed, reflected and dispersed. Therefore, the spectrum recorded by the antenna-receiver is a superposition of all harmonic components and may have a changed shape and distribution in relation to the source signal.

In order to determine which element in the robot is responsible for intensifying the level of disturbances, an analysis was carried out using a spectrum analyzer and near-field probes. By scanning the locations in which the individual robot sub-circuits are located, it was found that the propulsion system radiates the most intensively. Despite the fact that the harmonic components change their position dynamically in the spectrogram, it can be seen that the engine and its control system have the characteristic frequency bands associated with the spectrum during measurements in the chamber (Fig. 13).

To get the answer what is the cause of this phenomenon, a time analysis of current and voltage waveforms was performed for the three characteristic speeds of the robot movement. During the tests, the waveform of the current drawn from the battery, and the voltage at the motor terminals were analyzed. The speed of the wheels is regulated by changing the voltage supplying the motor using PWM modulation. Although the current measured at the motor terminals is continuous and does not drop to zero, even for the smallest programmed speed, some pulse components can be observed. They are conditioned by the process of switching of the transistors in the bridge (Fig. 14).

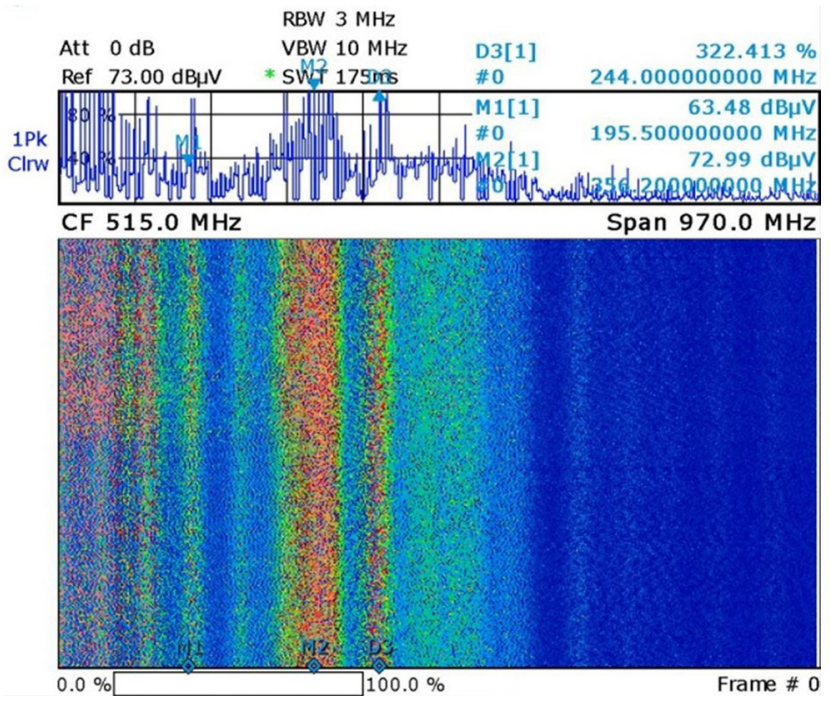

Fig. 13 Spectrum of disturbances generated near one of the motors in the robot 


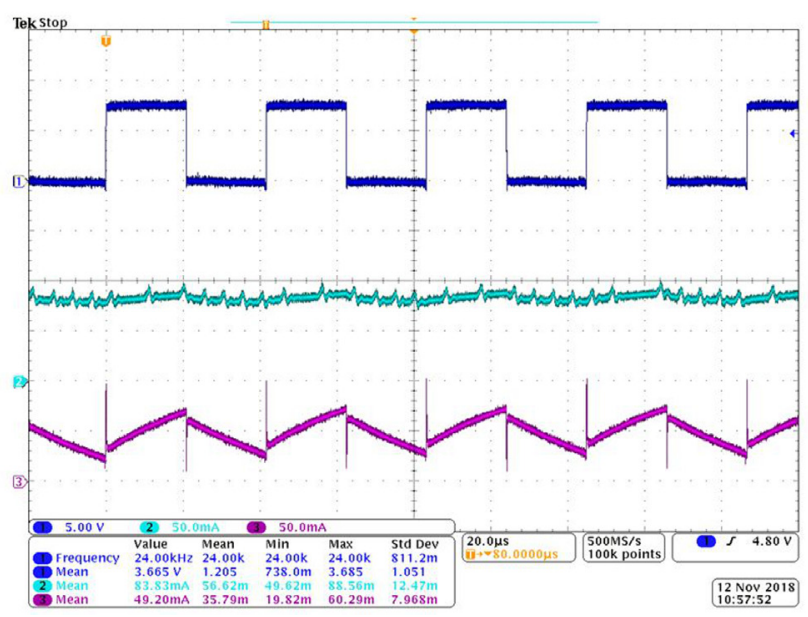

Fig. 14 Voltage and current drawn from the battery at the motor terminals at the speed of $0.5 \mathrm{~m} / \mathrm{s}$

These components cause the generation of disturbances in the frequency band from $150 \mathrm{MHz}$ to $400 \mathrm{MHz}$ (Figs. 7, 8). In the case of the motor running at the highest speed, the spectrum of harmonic components increases significantly and the harmonics also increase their level.

In the waveform of both the current drawn and the voltage, one can notice a peek with a much greater value than that caused by the voltage regulation (Figs. 15, 16). The pulse character of the waveform with oscillation decaying at the frequency of $\sim 90 \mathrm{MHz}$ is the consequence of commutation of current in the commutator of the motor. The transition of brushes between successive sections results in the generation of impulse disturbances with values several times higher than the rated current and voltage. This has its consequence in the increased level of the generated disturbances at the highest speed of the robot movement.

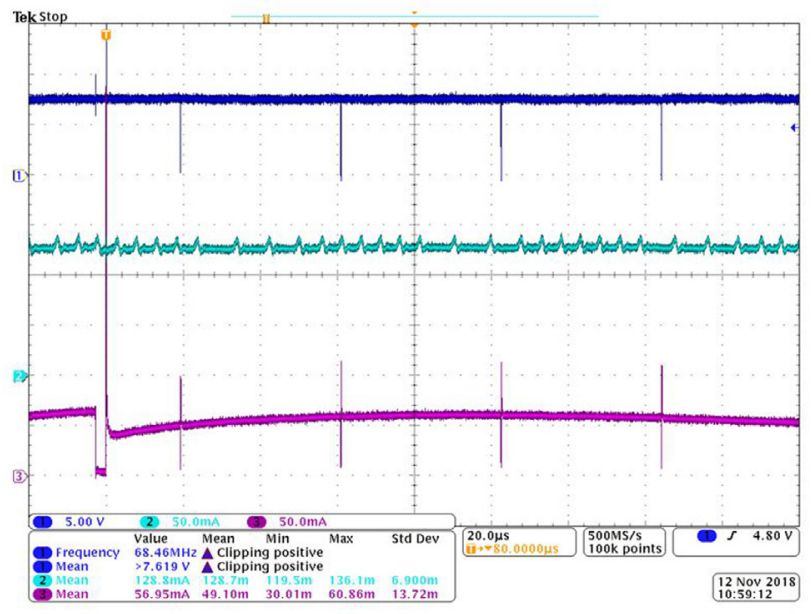

Fig. 15 Voltage and current drawn from the battery at the motor terminals at the speed of $1 \mathrm{~m} / \mathrm{s}$
At lower speeds, the effect of commutation of energy on the commutator has less impact on the level of disturbances.

\section{Conclusions}

The conducted analysis and research show that the issue of EMC compatibility of mobile robots is an important factor that should be taken into account by engineers. It may influence the correct work of the system where multiple radio communication systems are used for navigation and for communication between individual robots in a group or with the master control unit.

The tests that a mobile robot should undergo in the aspects of electromagnetic compatibility are strictly dependent on its working environment. The environment determines the allowed level of disturbances generated by the robot as well as the class of potential electromagnetic disturbances that may affect its operation. The measurements of the disturbance emission for the prototype robot were carried out in accordance with the requirements of the general standard EN 61000-6-3. It is more restrictive in the field of emission of electromagnetic disturbances than the standard which defines the requirements for the industrial environment, i.e. EN 61000-6-4. Despite the fact that the tested robot can be included in the class of miniature elements, the disturbances it generates are close to the permitted limit values, as the results of the tests shown. The disturbances are mainly a consequence of a non-optimal work of the drive unit. The classic commutator motors loaded with the rated power are the source of electromagnetic disturbances of significant levels that can be reduced by introducing anti-interference elements into the robot's drive unit.

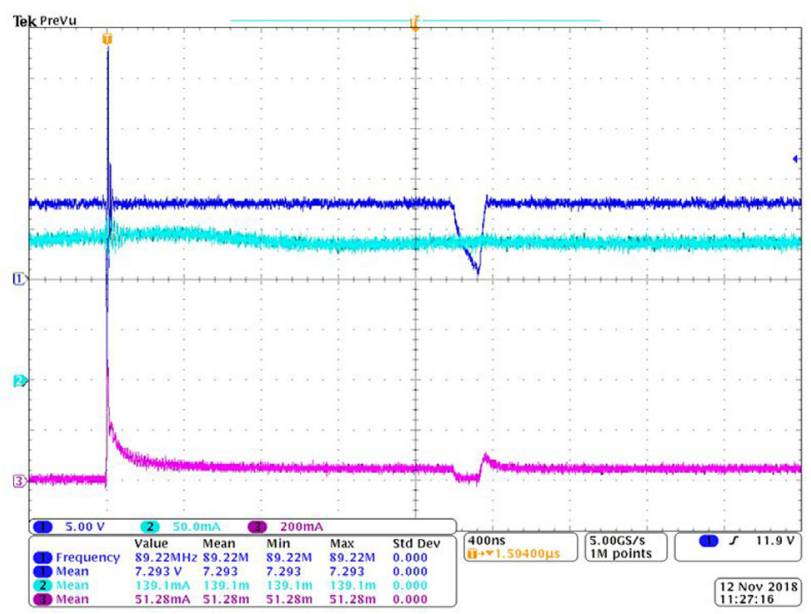

Fig. 16 Enlarged portion of the waveform of voltage and current drawn from the battery at the motor terminals at the speed $1 \mathrm{~m} / \mathrm{s}$ 


\section{Acknowledgement}

Results of Grant No. PBS1/A3/3/2012 from Polish National Centre for Research and Development as well as Statutory Activity of Rzeszow University of Technology were applied in this work. The work was developed by using equipment purchased in Operational Program

\section{References}

[1] Finkenzeller, K. "RFID Handbook: Fundamentals and Applications in Contactless Smart Cards, Radio Frequency Identification and near-Field Communication", 3rd ed., John Wiley \& Sons, Chichester, UK, 2010.

https://doi.org/10.1002/9780470665121

[2] Willis, S., Helal, S. "RFID information grid for blind navigation and wayfinding", In: Ninth IEEE International Symposium on Wearable Computers (ISWC'05), Osaka, Japan, 2005, pp. 34-37. https://doi.org/10.1109/ISWC.2005.46

[3] Ni, L. M., Liu, Y., Lau, Y. C., Patil, A. P. "LANDMARC: Indoor Location Sensing Using Active RFID", Wireless Networks, 10(6), pp. 701-710, 2004.

https://doi.org/10.1023/B:WINE.0000044029.06344.dd

[4] Subramanian, S. P., Sommer, J., Schmitt, S., Rosenstiel, W. "RIL - reliable RFID based indoor localization for pedestrians", In: 2008 16th International Conference on Software, Telecommunications and Computer Networks, Split, Croatia, 2008, pp. 218-222.

https://doi.org/10.1109/SOFTCOM.2008.4669483

[5] Yao, C., Hsia, W. "An Indoor Positioning System Based on the Dual-Channel Passive RFID Technology", IEEE Sensors Journal, 18(11), pp. 4654-4663, 2018.

https://doi.org/10.1109/JSEN.2018.2828044

[6] Pomárico-Franquiz, J. J., Shmaliy, Y. S. "Accurate Self-Localization in RFID Tag Information Grids Using FIR Filtering", IEEE Transactions on Industrial Informatics, 10(2), pp. 1317-1326, 2014. https://doi.org/10.1109/TII.2014.2310952

[7] Soltani, M. M., Motamedi, A., Hammad, A. "Enhancing Clusterbased RFID Tag Localization Using Artificial Neural Networks and Virtual Reference Tags", In: International Conference on Indoor Positioning and Indoor Navigation, Montbeliard-Belfort, France, 2013, pp. 1-10.

https://doi.org/10.1109/IPIN.2013.6817886

[8] Yu, H., Chen, J., Hsiang, T. "Design and Implementation of a RealTime Object Location System Based on Passive RFID Tags", IEEE Sensors Journal, 15(9), pp. 5015-5023, 2015. https://doi.org/10.1109/JSEN.2015.2432452

[9] Zhou, J., Shi, J. "RFID localization algorithms and applications - a review", Journal of Intelligent Manufacturing, 20, pp. 695-707, 2009. https://doi.org/10.1007/s10845-008-0158-5

[10] Saab, S. S., Nakad, Z. S. "A Standalone RFID Indoor Positioning System Using Passive Tags", IEEE Transactions on Industrial Electronics, 58(5), pp. 1961-1970, 2011. https://doi.org/10.1109/TIE.2010.2055774
Development of Eastern Poland 2007-2013, Priority Axis I Modern Economics, Activity I.3 Supporting Innovation under Grant No. POPW.01.03.00-18-012/09-00 as well as Program of Development of Podkarpacie Province of European Regional Development Fund under Grant No. UDA-RPPK.01.03.00-18-003/10-00.

[11] Park, S., Lee, H. "Self-Recognition of Vehicle Position Using UHF Passive RFID Tags", IEEE Transactions on Industrial Electronics, 60(1), pp. 226-234, 2013. https://doi.org/10.1109/TIE.2012.2185018

[12] Deyle, T., Nguyen, H., Reynolds, M., Kemp, C. "RFID-Guided Robots for Pervasive Automation", IEEE Pervasive Computing, 9(2), pp. 37-45, 2010. https://doi.org/10.1109/MPRV.2010.17

[13] Boccadoro, M., Martinelli, F., Pagnotelli, S. "Constrained and quantized Kalman filtering for an RFID robot localization problem", Autonomous Robots, 29(3-4), pp. 235-251, 2010. https://doi.org/10.1007/s10514-010-9194-z

[14] DiGiampaolo, E., Martinelli, F. "Mobile Robot Localization Using the Phase of Passive UHF RFID Signals", IEEE Transactions on Industrial Electronics, 61(1), pp. 365-376, 2014. https://oi.org/10.1109/TIE.2013.2248333

[15] Gueaieb, W., Suruz Miah, M. "An Intelligent Mobile Robot Navigation Technique Using RFID Technology", IEEE Transactions on Instrumentation and Measurement, 57(9), pp. 1908-1917, 2008 https://oi.org/10.1109/TIM.2008.919902

[16] Han, S., Lim, H., Lee, J. "An Efficient Localization Scheme for a Differential-Driving Mobile Robot Based on RFID System", IEEE Transactions on Industrial Electronics, 54(6), pp. 3362-3369, 2007. https://doi.org/10.1109/TIE.2007.906134

[17] Choi, B., Lee, J., Lee J., Park, K. "A Hierarchical Algorithm for Indoor Mobile Robot Localization Using RFID Sensor Fusion", IEEE Transactions on Industrial Electronics, 58(6), pp. 2226 $2235,2011$. https://doi.org/10.1109/TIE.2011.2109330

[18] DiGiampaolo, E., Martinelli, F. "A Passive UHF-RFID System for the Localization of an Indoor Autonomous Vehicle", IEEE Transactions on Industrial Electronics, 59(10), pp. 3961-3970, 2012. https://doi.org/10.1109/TIE.2011.2173091

[19] Papapostolou, A., Chaouchi, H. "RFID-assisted indoor localization and the impact of interference on its performance", Journal of Network and Computer Applications, 34(3), pp. 902-913, 2011. https://doi.org/10.1016/j.jnca.2010.04.009

[20] International Organization for Standardization "ISO/IEC 15693 3:2009 Identification cards - Contactless integrated circuit cards - Vicinity cards, Part 3: Anticollision and transmission protocol", 2009. [online] Available at: https://www.iso.org/standard/43467. html [Accessed: 10 October 2018] 
[21] International Organization for Standardization "ISO/IEC 144433:2018, Identification cards - Contactless integrated circuit cards Proximity cards", 2018. [online] Available at: https://www.iso.org/ standard/73598.html [Accessed: 10 October 2018]

[22] Hubacz, M., Pawlowicz, B., Trybus, B. "Exploring aSurface Using RFID Grid and Group of Mobile Robots, In: Szewczyk, R., Zieliński, C., Kaliczyńska, M. (eds.) Automation 2018: AUTOMATION 2018. Advances in Intelligent Systems and Computing, Vol. 743, Springer, Cham, Switzerland, 2018, pp. 490-499.

https://doi.org/10.1007/978-3-319-77179-3_46

[23] Directive 2014/30/EU of the European Parliament and of the Council of 26 February 2014 on the harmonisation of the laws of the Member States relating to electromagnetic compatibility. [online] Available at: https://ec.europa.eu/growth/single-market/ european-standards/harmonised-standards/electromagneticcompatibility_en [Accessed: 10 October 2018]

[24] Directive 2014/53/EU of the European Parliament and of the Council of 16 April 2014 on the harmonisation of the laws of the Member States relating to the making available on the market of radio equipment. [online] Available at: https://ec.europa.eu/ growth/single-market/european-standards/harmonised-standards/ red_en [Accessed: 10 October 2018]
[25] International Electrotechnical Commission "IEC 61000-63:2006+AMD1:2010 CSV Consolidated version, Electromagnetic Compatibility - Part 6-3: Generic Standards - Emission Standard For Residential, Commercial And Light-industrial Environments", 2011. [online] Available at: https://webstore.iec.ch/publication/4247 [Accessed: 10 October 2018]

[26] Jankowski-Mihułowicz, P., Kalita, W., Pawłowicz, B. "Problem of dynamic change of tags location in anticollision RFID systems", Microelectronics Reliability, 48(6), pp. 911-918, 2008. https://doi.org/10.1016/j.microrel.2008.03.006

[27] Jankowski-Mihułowicz, P., Węglarski, M. "Determination of 3-Dimentional Interrogation Zone in Anti-collision RFID Systems with Inductive Coupling by Using Monte Carlo Method", Acta Physica Polonica A, 121(4), pp. 936-940, 2012. https://doi.org/10.12693/APhysPolA.121.936 